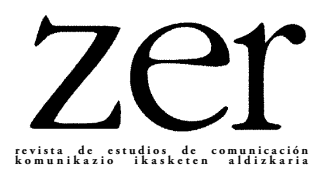

\title{
¿Quiénes hablan en las noticias? Desequilibrio de género en las fuentes informativas de la prensa de proximidad
}

\author{
Who speaks on the news? \\ Gender imbalance in local press sources
}

Cristina Prieto Sánchez ${ }^{\star}$

\begin{abstract}
RESUMEN: El objetivo de este artículo es determinar si mujeres y hombres participan de manera equilibrada en los contenidos que los medios ofrecen a la audiencia. El análisis de los datos constata que ni las voces recogidas en las noticias ni sus posiciones dentro de las mismas alcanzan la paridad. Las mujeres ocupan lugares poco relevantes y desaparecen de los informativos locales en el tránsito de las convocatorias a las noticias elaboradas. Este artículo presenta los resultados de un estudio de caso realizado sobre los medios de Granada para el que se han evaluado 1.586 informaciones y 184 convocatorias de agenda.
\end{abstract}

PALABRAS CLAVE: Medios de comunicación, comunicación local, género, fuentes informativas.

LABURPENA: Artikulu honen xedea da hedabideek ikusleei eskaintzen dizkieten edukietan emakumeen eta gizonen parte-hartzea orekatua den ala ez zehaztea. Datuak aztertu ondoren, ondoriozta daiteke albisteetan jasotako ahotsek ez eta ahots horien posizioak ere ez dutela parekidetasuna erdiesten. Emakumeek garrantzia txikiko posizioak izaten dituzte, eta tokiko albistegietatik desagertzen dira albiste xehatuagoak ematen direnean. Artikulu honetan, Granadako hedabideei buruzko kasu-ikerketa baten emaitzak jasotzen dira; bertan, 1.586 informazio eta 184 agenda-deialdi aztertu dira.

HITZ-GAKOAK: Hedabideak, tokiko komunikazioa, generoa, informazio-iturriak. 


\begin{abstract}
The objective of this article is to determine if women and men participate in a balanced way in the contents offered by the media to the audience. The analysis of the data shows that neither the voices collected in the news nor their positions within them reach parity. Women are generally relegated to scarcely relevant places and, in local news, they often disappear altogether in the course towards the final piece of news. This article presents the results of a case study conducted on the media of Granada for which 1,586 news reports and 187 agenda press calls were evaluated.
\end{abstract}

KEYWORDS: Mass media, local communication, gender, information sources.

* Correspondencia a: Cristina Prieto Sánchez. C/Ancha de Gracia, 5-6 A (18002 Granada).cpcristinaprieto@gmail.com

http://orcid.org/0000-0002-9151-081X

Cómo citar: Prieto Sánchez, Cristina. 2018. ¿Quiénes hablan en las noticias? Desequilibrio de género en las fuentes informativas de la prensa de proximidad. Zer. 23(45). 161-184. https://doi.org/10.1387/zer.

Recibido: 00 octubre 2018; aceptado: 00 octubre 2018

1137-1102 y 1989-631X / (C) 2018 UPV/EHU 


\section{Introducción}

El pasado 8 de marzo marcó un punto de inflexión en las reclamaciones de las mujeres, sin embargo, continúan relegadas en las informaciones que periódicos, emisoras de radio y televisiones lanzan a diario a su audiencia, tanto a nivel nacional como local. En ocasiones, se habla de lo que hacen, lo que dicen, lo que piensan pero ¿quiénes hablan?

El origen de este estudio surge como respuesta a las inquietudes de un grupo de periodistas e investigadoras granadinas ${ }^{1}$ interesadas por conocer la realidad de los datos sobre el desequilibrio de género en las noticias de la provincia en cuanto al número de declaraciones femeninas y masculinas en las informaciones. Como parte de la audiencia, intuíamos que los porcentajes no representan la realidad social que nos rodea y, como profesionales de los medios, queríamos averiguar dónde se producen esos desequilibrios y si la visión androcéntrica de las noticias alcanzaba también a aquellas fuentes informativas en cuya cúspide se sitúan mujeres. Constituye pues, una buena colaboración entre la profesión y la academia para explorar, desde distintos ángulos, la realidad de la información de proximidad, sus prácticas, la agenda con la que se construye el mosaico informativo diario y la realidad de sus protagonistas.

Las trece periodistas que hemos participado en la elaboración de este estudio hemos accedido a las informaciones analizadas sin privilegios, como parte de la audiencia (excepto para las noticias de agencia que sólo llegan a los abonados pero con cuyo servicio hemos podido contar a través de compañeras que trabajan en gabinetes de comunicación y tienen acceso a estos contenidos) por lo que podemos tildar esta investigación de una auditoría ciudadana de la información en Granada. Para ello hemos contado con la experiencia de dos de las componentes del equipo (entre las que se encuentra la autora de este artículo) que participaron en 2015 en el Monitoreo Global de Medios (GMMP) cuya metodología de estudio hemos tomado como base aunque con algunas modificaciones para adaptarla a nuestro objeto de estudio.

\section{Una aproximación teórica a los estudios de género y medios de comunicación}

Con la consolidación de la democracia en España, el marco legal que proporcionó en 1978 la Constitución Española y la Ley de Igualdad de 2007 aprobada por el go-

\footnotetext{
${ }^{1}$ Las profesionales que han participado en el trabajo de campo de este estudio son: María José Anguita Escolano, Manuela Cobo Maeso, Lola Fernández Palenzuela, Ana Fuentes Prior, Mónica García García, Noelia Jiménez García, Ángela Jiménez Maldonado, Marisa Molina Carbonell, Silvia Muñoz Pérez, Lola Prieto Moraleda, Luz Sánchez Gámez, Esther Sanz Urcia y la autora de este artículo.
} 
bierno de José Luis Rodríguez Zapatero, parecía que las mujeres disfrutaban ya de un marco legal suficientemente desarrollado para fijar su posición por derecho en todos los ámbitos de nuestra sociedad. Sin embargo, la realidad nos devuelve cada día la otra cara de la moneda y nos demuestra que lo expuesto con claridad en estos textos legales no es así en la práctica y que aún queda un largo camino por recorrer para la consecución de una sociedad igualitaria. En este proceso, los medios de comunicación tienen un papel fundamental en cuanto que son transmisores de los valores sociales «que acaban conformando el ideario de nuestro modelo social y de nuestras actitudes» (Beltrán, 2014: 720).

Para entender mejor nuestro entorno, los estudios de género constituyen una de las mejores aportaciones (Papí, 2003), especialmente para el tema que nos ocupa, en tanto que orientados a la investigación del papel de las mujeres en los medios de comunicación y la publicidad, bien como parte de la estructura organizativa de las empresas periodísticas o como protagonistas de las informaciones presentadas a la opinión pública por periódicos, emisoras de radio, formatos digitales, revistas, televisiones o agendas de noticias. En la década de los 70, Courtney y Lockeretz (1971) abordaron los roles con los que las mujeres trabajadoras estaban representadas en la publicidad, aspecto que también trataron Culley y Bennett (1976) quienes centraron su investigación en los anuncios.

En la década de los 80, Tan (1982) y Zemach y Cohen (1986) introdujeron la idea de que los estereotipos están relacionados con el consumo televisivo y Litcher y Rothman (1986) se centraron en la proporción de mujeres como presentadoras de televisión. Tuchman (1983) determinó el papel de las profesionales de la comunicación dentro de la estructura productiva de los medios, Karpf (1980) colocó en su punto de mira a los formatos radiofónicos y Gallagher apuntaba que la desigualdad de oportunidades entre mujeres y hombres en el campo de la comunicación en aquellos momentos, estaba en el dominio de los varones por lo que «el personal femenino de los medios de comunicación social ocupa una posición claramente desventajosa en comparación con la de sus colegas masculinos» (Gallagher, 1983: 40).

El final de pasado siglo también dejó investigaciones relevantes sobre el género y los medios de comunicación como el de Pearson, Turner y Todd-Mancillas (1993) «Comunicación y género» y el de Trancart (1999) Les femmesabsentes de l'information. Quand les chiffresparlent.

En España, Franquet (1982a), Fagoaga y Secanella (1984), Comas (1988) y Gallego et al. (1998) realizaron importantes aportaciones a la materia y señalaron el desequilibrio entre las mujeres pertenecientes a las cúpulas directivas de los medios y sus compañeros varones. Los diversos estudios realizados a principios del presente siglo han puesto de manifiesto tanto la invisibilidad de la mujer en los medios como 
la influencia de estereotipos masculinos en los contenidos (García de Cortázar, M. y García de León M.A., 2000; Canel, M.J., 2000; Humanes, M.L., 2003; Soriano, J., Cantón, M.J. y Díez, M., 2005 y Blanco Castilla, E., 2005, entre otros).

En los últimos años, las líneas de investigación sobre el género y los medios de comunicación se han ampliado y han puesto el foco en la relación existente entre la ausencia de mujeres en puestos de responsabilidad dentro de las estructuras orgánicas de los medios de comunicación y los contenidos informativos que continúan relegando los temas de género y las noticias con fuentes femeninas a lugares poco destacados dentro de cada uno de los formatos. Es destacable, en este aspecto, el trabajo realizado por la Alianza Global sobre Medios y Género (GAMAG) integrada en la red Red UniTWIN de la Unesco que tiene como objetivos incrementar la participación y acceso de las mujeres a la toma de decisiones en los medios y promover una representación equilibrada y no estereotipada en las noticias.

El estudio Global Report on the Status of Women in the News Media de la International Women's Media Foundation (IWMF) realizado en 2011 dejaba al descubierto la desigual relación de género en las redacciones ya que de las 170.000 personas empleadas en periodismo, los varones representaban casi dos tercios del total $(64,9 \%)$ frente al tercio de las mujeres $(35,1 \%)$. La investigación no dejaba datos alentadores para España donde la representación de mujeres en los cargos de alto nivel de dirección representaba sólo el 17,6\% y esta baja presencia indica su falta de poder en los medios de comunicación.

\section{La mirada androcéntrica de las noticias}

Los medios de comunicación juegan un papel esencial como transmisores de valores y como agentes de cambio social porque son un poderoso instrumento para modelar la opinión pública, «no sólo actúan como notarios de la realidad, sino que la construyen y la interpretan» (Moreno, 2001: 158). Pueden erigirse como motor de cambio o como rémora y esta dualidad es lo que les convierte en un foro público tan importante porque, de una parte «reproducen las bases cognitivas de la sociedad y en parte son poderosos instrumentos para poder propiciar el cambio social» (Gallego, 2010: 6). Y en este punto, cabría preguntarse ¿quiénes dirigen esos medios de comunicación? La respuesta es obvia, los hombres en su gran mayoría. Ellos continúan dominando el espacio público, creando opinión y enfocando las noticias desde una perspectiva androcéntrica.

Un cúmulo de circunstancias, algunas debidas a las malas prácticas por parte de los profesionales de la información y la comunicación y otras de difícil solución porque atañen directamente a las empresas propietarias, han favorecido que los medios se resistan a dar cuenta de los cambios sociales y persistan en un enfoque androcén- 
trico «restringido a una parte de la población, varones adultos de pueblos y clases dominantes que actúan en los escenarios del poder, que margina a la mayoría de mujeres y hombres como si sus aportaciones fueran insignificantes para el buen funcionamiento de la democracia» (Moreno y Simelio, 2011).

Entre las malas prácticas nos encontramos con un exceso de fuentes institucionales que coloca el foco de atención en sus máximos responsables (hombres), el seguimiento constante de la agenda política que determina un amplio porcentaje de los contenidos informativos y aleja a los medios de la diversidad social y las rutinas que las mujeres periodistas han aceptado al integrarse en las redacciones (Franquet, 1982b; Altés y Gallego, 2004) establecidas desde una óptica exclusivamente masculina con el objetivo de "ser una de ellos» (Melin-Higgins y Dejerf Pierre, 1998) y no ver comprometida la posibilidad de ascender en la estructura organizativa del medio.

Las causas relacionadas con las empresas apuntan a la reducción de las redacciones hasta límites incompatibles con la calidad de los contenidos, la presión ejercida por los grandes grupos multimedia que han convertido la información en productos que pretenden venderse con criterios de marketing y a la precariedad laboral de los y las periodistas.

Aun así, todas estas circunstancias no eximen al conjunto de profesionales de los medios de comunicación de aplicar la perspectiva de género en los contenidos periodísticos para que comiencen a ser tipificados como un posible "valor noticia» (Gallego, 2003):

La dimensión de género - es decir: aquellos rasgos que afectan a las personas por razón de pertenecer a un género, y que creemos son significativos informativamente-, no está tipificada como un posible 'valor noticia', de tal manera que esta perspectiva permanece ignorada y oculta en la mayoría de las informaciones, y tan solo hace acto de presencia cuando es la propia fuente la que incorpora la significación de género.

Es necesario que a los puestos de dirección accedan periodistas y comunicadoras con perspectiva de género para imprimir a los contenidos una visión de la realidad más cercana a la sociedad paritaria y democrática a la que aspiramos. Serán ellas, desde los estamentos jerárquicos de las redacciones donde se deciden los temas de cobertura y la agenda diaria, las que podrán evitar que los contenidos con temas de género se queden en «los ámbitos periféricos de la escena pública» (Gallego, 2003) y cuenten con más posibilidades de ser tratados. Aún hoy, las redacciones «están copadas ya no por los varones sino por los valores androcéntricos; las condiciones laborales desalientan las reivindicaciones, y el conjunto de todo ello permite la defensa y la legitimación del dominio» (Vera Balanza, 2009: 70). 
Las cifras ponen de manifiesto que el número de mujeres en puestos directivos es muy bajo pese a que la profesión está, en términos globales, feminizada. En 2009, el porcentaje para ellas se situaba en el 14,7 frente al 85,3 de los hombres a nivel nacional y tomando como base de este estudio la Agenda de la Comunicación del año 2009 publicada por el Gobierno de España y las 103 cabeceras nacionales de prensa diaria (Caro, García-Gordillo y Bezunartea, 2010). Este estudio aventuraba la hipótesis de que a mayor número de mujeres directivas y mayor número de directoras se produce un aumento de lectoras femeninas y un cambio en los valores noticiables, tal y como proponían las investigaciones feministas en comunicación y, particularmente, Van Zoonen (1994).

Los datos más recientes persisten en la situación desfavorable de las mujeres en el sector de la comunicación. El Informe Anual de la Profesión Periodística en España 2017, editado por la Asociación de la Prensa de Madrid, constata que la precariedad laboral se ceba en las mujeres con un 64\% de paro, frente al 36\% de los varones.

\section{Las fuentes informativas como indicador de calidad}

En el ámbito profesional es sabido que un periodista vale lo que pesa su agenda y que las fuentes y su nivel de conocimiento o cercanía al tema tratado determinan el valor de la información. En estos momentos, los y las periodistas son, cada vez en mayor medida, procesadores de información porque están abandonando su tarea de «escrutinio crítico sobre las élites políticas y económicas de la sociedad» (Casero, 2012) con el objetivo de defender los intereses de los ciudadanos y alertar de los posibles abusos de los poderosos. Por eso, esta tarea del periodismo le configura como un Cuarto Poder y los periodistas se convierten en el perro guardián (watchdog) de la democracia. Sin embargo, muchos profesionales se están entregando, exclusivamente, a la gestión de los contenidos externos que reciben (Rodríguez, Enguix, Rojas y García-Gordillo, 2015).

La proliferación de gabinetes y agencias de comunicación en instituciones y empresas, con periodistas profesionales, inundan a diario las redacciones de notas de prensa redactadas y estructuradas perfectamente a las que, en muchas ocasiones, se acompañan de cortes de sonido, totales de vídeo o fotografias preparadas para ser publicadas. Como señala Schudson, estos «paraperiodistas» no dejan tiempo a los profesionales de los medios para hablar con sus contactos y cuidar sus fuentes por lo que seguir temas propios se ha convertido en un lujo (Schudson, 2003: 3) En las redacciones, las exiguas plantillas y el fast journalism espoleado por los medios digitales que actualizan constantemente las noticias, hacen el resto. El resultado son contenidos de baja calidad, carentes de profundidad, en los que las fuentes redactan la noticia, la protagonizan y dan su versión sin ningún otro elemento que ofrezca a la audiencia el necesario contraste para formarse una visión crítica del hecho referenciado. 
Esta situación es más preocupante en la prensa de proximidad. Los medios locales son los referentes de lo que acontece en el espacio geográfico más inmediato de la audiencia que se informa a través de ellos porque los receptores identifican los escenarios y porque las informaciones les afectan directamente. Si se delega en las fuentes la selección de temas que aparecen en los distintos soportes, el medio no está respondiendo al ritmo vital de la sociedad y los periodistas pasan a estar más atentos a las noticias de los políticos que a sus acciones de gobierno (García-Gordillo, Bezunartea, Rodríguez y Sánchez, 2014).

El creciente número de informaciones que presentan como protagonistas a instituciones, organizaciones políticas, empresas, datos, cosas o entes, están deshumanizando la información y alejando el foco de la ciudadanía plural. «Este retroceso en el enfoque de los protagonistas humanos afecta principalmente a las mujeres y a los colectivos que forman parte de la sociedad civil. Contrariamente, la atención hacia los varones identificados ha permanecido estable» (Moreno, Simelio, Rovetto y Buitrago, 2007).

Precisamente, el tratamiento informativo de las mujeres en los medios es el objetivo principal del Proyecto Monitoreo Global de Medios (GMMP en sus siglas en inglés), la investigación mundial más amplia y de mayor trayectoria que aborda el tema de género en los medios de comunicación. Se inició en 1995, como resultado de los acuerdos alcanzados en la Conferencia Internacional de Beijing, cuando voluntarios y voluntarias en 71 países de todo el mundo (en 2015 han participado 114) monitorearon la presencia de las mujeres en los espacios informativos de radio, televisión y prensa escrita nacionales. La investigación reveló que sólo 17\% de los sujetos de las noticias - esto es, las personas entrevistadas o sobre quienes tratan las noticas- fueron mujeres.

Cada cinco años se realiza un nuevo estudio y, aunque las cifras de representación de las mujeres han evolucionado hacia porcentajes más positivos, la progresión es muy lenta y en los últimos cinco años se ha estancado, contradiciendo las propias dinámicas sociales que acontecen respecto a la presencia y la participación de las mujeres en el mundo. Del 17\% del primer estudio en 1995 al 24\% en el último de 2015 - la misma cifra que se alcanzó en 2010 - puede decirse que la representación del $50 \%$ de la población mundial no ha estado acompasada con los cambios sociales que se han producido en estos últimos 20 años. Margaret Gallagher alerta sobre la situación en el prólogo del informe de 2015:

La realidad es que el tema de los medios languidece al margen de la mayor parte de las agendas políticas que promueven la igualdad de género. A 20 años de Beijing, las transformaciones tecnológicas han contribuido a que los medios y la comunicación se atrincheren todavía más en relaciones de poder de género y en su negociación. 
En España, los datos son algo más alentadores pero se sitúan lejos de la paridad y de la realidad. Las mujeres comprenden sólo el $28 \%$ del total de sujetos y fuentes en las noticias de prensa y los informativos de radio y televisión y el 33\% en los diarios digitales y Twitter. Este porcentaje es ligeramente superior al de la media europea que es de $26 \%$ y $25 \%$, respectivamente. También significa un pequeño avance respecto a los datos del estudio de 2010, donde las mujeres en las noticias de prensa, radio y televisión eran el 23\% del total.

El trabajo del Monitoreo Global de Medios se realiza sobre las noticias difundidas durante una única jornada (en el caso de 2015 se eligió como fecha el 25 de marzo) y en distintos soportes (diarios impresos, radios, televisiones, diarios digitales y Twitter). La evaluación se realiza sobre medios y espacios informativos de ámbito nacional por lo que quedan al margen los medios de ámbito regional y local. Entendemos que es en este marco donde se puede observar, por comparación y contraste, si los medios son receptivos a los cambios producidos.

\section{Objetivo e hipótesis}

El objetivo principal de este estudio de caso es determinar las fuentes utilizadas por los medios de comunicación de Granada y analizar la relevancia otorgada a las mismas para conocer si el género condiciona la posición que ocupan dentro de cada de una de las informaciones difundidas. Sustentada en nuestra percepción como consumidoras de medios de comunicación y profesionales de los mismos, nuestra hipótesis de partida apunta que las voces femeninas como fuentes informativas tienen menor peso en la información local que las voces masculinas. Al mismo tiempo, el análisis de las noticias permitía además conocer si los medios de comunicación recogían en sus informaciones identidades de género distintas a la fórmula binaria femenino/masculino acercándose de esta manera a la realidad social actual.

Diseñada esta línea de trabajo, nos planteamos las siguientes preguntas de investigación: ¿qué temas informativos cuentan con fuentes femeninas?, si la práctica profesional otorga más valor noticiable a los máximos representantes de las instituciones ¿aparecerán con más frecuencia las declaraciones femeninas en instituciones u organismos que cuentan con mujeres en la cúspide de su organigrama?, ¿marca la agenda informativa distribuida por las agencias de noticias la posición de las declaraciones femeninas y masculinas en las informaciones que difunden los medios? ¿Se da voz a otras identidades de género presentes en la sociedad? 


\section{Metodología}

La metodología mixta y descriptiva diseñada para abordar estas cuestiones y encontrar respuesta a nuestras preguntas nos llevó, en primer lugar, a seleccionar los medios de comunicación que iban a ser objeto de nuestro estudio y el período de tiempo destinado al seguimiento de sus contenidos. Realizar coberturas diarias de la agenda informativa local, contar con una plantilla estable de profesionales de la información y estar presentes de forma continuada en el panorama informativo provincial fue el criterio acordado. Estas condiciones las cumplían cinco emisoras de radio - Radio Nacional de España, Onda Cero, Cadena Cope, Canal Sur Radio y Cadena Ser-, dos televisiones - Canal Sur TV y TG7-, tres diarios digitales - Ahora Granada, Granada Digital y El Independiente de Granada-, dos diarios impresos — Ideal y Granada Hoy - y dos agencias de noticias — Europa Press y Agencia EFE-.

El estudio se llevó a cabo durante dos semanas completas, de lunes a domingo; la primera del 21 al 27 de mayo y la segunda del 4 al 10 de junio. Se descartó la semana del 28 de mayo al 3 de junio por la celebración de la Feria del Corpus en Granada y la alteración que en estas fechas registra la información local en su conjunto. Para tener una visión amplia de los contenidos informativos ofrecidos a la audiencia por los doce medios seleccionados y las dos agencias de noticias se analizaron, en los formatos audiovisuales, los programas informativos completos destinados a las noticias locales en franjas de mañana y mediodía para las radios y de mediodía y noche para las televisiones locales.

El análisis de las noticias en los medios digitales se realizó sobre todas las informaciones locales subidas a la web durante una jornada completa y en los periódicos en papel se evaluaron todas las noticias locales en cada una de las secciones del número seleccionado.

Los contenidos distribuidos por las agencias de noticias se sometieron a un doble análisis. Por un parte los despachos correspondientes a la cobertura de noticias locales enviadas a los abonados y, por otra, las convocatorias de agenda donde se recogen todas las citas informativas de la jornada. En todos los casos, las evaluaciones se realizaron, en cada medio, en días alternos.

Quedaron al margen de nuestro análisis las informaciones de servicio (tráfico, predicciones meteorológicas, anuncios por palabras...), los artículos de opinión, los editoriales, las columnas, los espacios de humor gráfico, los suplementos, los publirreportajes, las noticias patrocinadas dentro de los espacios informativos y los sumarios o 'llamadas' en las portadas de los diarios en papel porque su brevedad no podía aportar datos a nuestra investigación. 
Para conocer la procedencia de las fuentes informativas se diseñó una plantilla de trabajo para cada tipo de soporte (televisiones, radios, diarios digitales, diarios en papel, noticias de agencia y convocatorias de agencia) en la que se incluyeron los 17 sectores generadores de noticias en Granada (16 específicos más uno con la categoría de "otros» para aquellos contenidos de dificil inclusión en las anteriores). Además, se destinó un espacio para señalar el número de declaraciones textuales identificadas de cada noticia, el género ${ }^{2}$ de cada una y el lugar que ocupa dentro de la información.

En total, se han analizado 1.586 informaciones y 184 convocatorias de agenda con la siguiente distribución por formato:

\section{TABLA 1}

Medios y noticias analizadas

\begin{tabular}{|l|c|}
\hline \multicolumn{1}{|c|}{ Tipo de medio } & $\begin{array}{c}\text { Número de informaciones } \\
\text { analizadas }\end{array}$ \\
\hline Radios & 245 \\
\hline Diarios digitales & 388 \\
\hline Prensa diaria en papel & 610 \\
\hline Televisiones & 95 \\
\hline Noticias de agencias & 248 \\
\hline Convocatorias de agencias & 184 \\
\hline
\end{tabular}

Fuente: Elaboración propia.

\section{Resultados}

\subsection{Declaraciones de MUJERES y hOMbreS EN LAS NOTICIAS}

El análisis pormenorizado de las informaciones evaluadas confirma que las voces femeninas se escuchan o se leen en los medios de comunicación de Granada la mitad que las voces masculinas. De las 1.586 noticias evaluadas, el 41,60 contienen declaraciones realizadas por hombres (861 citas textuales) mientras que el 22,70 corres-

\footnotetext{
${ }^{2}$ La casilla destinada al género de cada noticia contempló la posibilidad de encontrar informaciones fuera del planteamiento binario hombre/mujer pero en los días analizados no se registró ninguna información de estas características por lo que en nuestras tablas no queda reflejado.
} 
ponden a mujeres (470 citas textuales) y el 35,70 tienen como fuentes informativas instituciones, organismos, empresas o entes, es decir, un origen no humano. Para la consideración de "declaración» sólo se han tenido en cuenta las frases textuales entrecomilladas y perfectamente identificadas con nombres, apellidos y cargo o responsabilidad que ostenta la persona que la realiza (para los medios escritos), los cortes de voz (para las radios) o los totales (para las televisiones).

El reconocimiento de las fuentes en la prensa de proximidad es esencial para contextualizar la noticia porque el espacio geográfico reducido en el que se sitúan otorgan cercanía y reconocimiento del trabajo realizado a la vez que ofrecen a los receptores la identificación de los escenarios (Rodríguez et al., 2015) y sus protagonistas. Por otro lado y en el caso de las mujeres, refuerzan su acción individual y reafirman su actividad pública, alejándolas del tratamiento como género o colectivo (Gallego, 2010). En este contexto, consideramos esencial para nuestra investigación delimitar a su sentido más restringido el concepto «declaración» ya que es habitual que en los textos periodísticos se incluyan referencias a lo que una persona dice pero sin incluir textualmente sus palabras ni la identificación completa de quien las pronuncia.

En el caso de las mujeres esta práctica supone una especie de «tutela informativa» (si se nos permite la expresión) porque aparece en los medios con declaraciones pasadas por el tamiz de quien realiza la información (periodista de la redacción del medio o responsable del gabinete de prensa que elabora la nota para distribuirla). La ausencia de las palabras textuales oculta el lenguaje utilizado por quien protagoniza la información y con ello puede diluir la idoneidad para la responsabilidad que ostenta invisibilizando y neutralizando el nivel intelectual y técnico, la capacitación o competencia de las protagonistas.

Aunque en el planteamiento metodológico diseñado para la realización de este estudio, como paso previo al trabajo de campo desarrollado con la evaluación de las noticias, ya se había tomado en consideración la posibilidad de encontrarnos con fuentes no sexuadas, el análisis de los datos nos ha dejado cifras no esperadas. El 35,70 de las informaciones presentan como fuente a instituciones, empresas, clubes de fútbol, sindicatos, tribunales... que «hacen», «dicen», "explican» o «aprueban» sin que aparezcan sus responsables. Esta práctica, cada vez más habitual en las redacciones, además de constituir una perversión del lenguaje (un ayuntamiento no dice, lo harán sus responsables) indica hasta qué punto la información se ha deshumanizado ${ }^{3}$ y cómo, en el reparto de papeles, ha entregado los de protagonistas a los varones y a las instituciones dejando de lado a las mujeres y a la ciudadanía plural (Moreno y Si-

3 Para ampliar este concepto véase la investigación $\mathrm{I}+\mathrm{D}+\mathrm{I}$ sobre «La representación de las relaciones entre mujeres $y$ hombres $y$ del recambio generacional en la prensa, de 1974 a 2004», financiada por el Instituto de la Mujer y liderada por Amparo Moreno Sardà. 
melio, 2011).La suma de las fuentes informativas de hombres y no humanas representa un 77,3 de la información local de Granada.

El reparto de las declaraciones masculinas y femeninas en las noticias locales ofrece datos relevantes. Del total de noticias con opiniones de hombres y mujeres recogidas de manera textual -1.190 en los diecisiete sectores generadores de noticias en los que se ha distribuido este estudio- las voces de ellos se encuentran en 757 noticias (un 63,61) mientras que las de ellas aparecen en 433 (un 36,38).

TABLA 2

Sectores informativos y porcentaje de noticias con declaraciones de hombres y mujeres

\begin{tabular}{|l|c|c|}
\hline \multicolumn{1}{|c|}{ Origen de las fuentes } & $\begin{array}{c}\text { Porcentaje de noticias } \\
\text { con declaraciones } \\
\text { masculinas }\end{array}$ & $\begin{array}{c}\text { Porcentaje de noticias } \\
\text { con declaraciones } \\
\text { femeninas }\end{array}$ \\
\hline Gobierno central & 3,03 & 0,50 \\
\hline Junta de Andalucía & 7,73 & 6,64 \\
\hline Diputación Provincial de Granada & 4,70 & 2,69 \\
\hline Ayuntamiento de Granada & 13,87 & 9,08 \\
\hline Universidad de Granada & 1,51 & 0,59 \\
\hline Partidos Políticos & 7,48 & 6,05 \\
\hline Organizaciones sindicales & 2,10 & 1,76 \\
\hline Tribunales & 2,77 & 0,92 \\
\hline Colectivos ciudadanos y ongs & 3,95 & 2,60 \\
\hline Bancos, cajas y entidades financieras & 0,42 & 0,00 \\
\hline Organizaciones empresariales & 1,76 & 0,76 \\
\hline Policía y Guardia Civil & 1,18 & 0,42 \\
\hline Colegios profesionales & 0,59 & 0,25 \\
\hline Clubes deportivos & 4,12 & 0,25 \\
\hline Artistas, escritores y profesionales liberales & 3,11 & 1,01 \\
\hline Fundaciones y asociaciones culturales & 0,92 & 0,92 \\
\hline Otros & 4,37 & 1,94 \\
\hline
\end{tabular}

Fuente: Elaboración propia.

La distribución por sectores generadores de noticias en los que se concentran las declaraciones confirma que los medios de comunicación de la prensa local de 
Granada se inclinan, mayormente, por dar voz a los varones. En temas como los relacionados con el Gobierno Central, los clubes deportivos o los contenidos culturales, las diferencias se triplican. Especialmente significativas resultan las cifras en las instituciones donde los hombres designados por los partidos políticos continúan copando los puestos de decisión y representación ante los medios de comunicación actuando como portavoces.

Especialmente llamativos son los porcentajes en la Junta de Andalucía y la Universidad de Granada que tienen como máximos responsables en Granada a dos mujeres, la Delegada del Gobierno andaluz y la Rectora, un posicionamiento institucional que no se traslada al tratamiento informativo de las noticias relacionadas con ambos sectores.

\subsection{Posiciones de las DeClaraciones}

En este estudio se han valorado también las posiciones que ocupan dentro de las noticias las declaraciones que realizan las distintas personas cualificadas ante los medios de comunicación, bien como portavoces o responsables de sus organizaciones o las administraciones a las que pertenecen, o bien como personas expertas sobre los temas tratados. Para valorar estas posiciones se han empleado criterios periodísticos. Es decir, las declaraciones que aparecen en primer término son las consideradas más importantes y las que aparecen con posterioridad se entienden como complementarias y, por ello, menos relevantes.

\section{TABLA 3}

Posición de las declaraciones de hombres y mujeres en los medios de comunicación de Granada. Porcentajes globales

\begin{tabular}{|c|c|c|c|c|c|}
\hline \multicolumn{2}{|c|}{ Declaraciones de hombres } & \multicolumn{3}{c|}{ Declaraciones de mujeres } \\
\hline $\begin{array}{c}1 .^{\mathrm{a}} \text { Fuente o } \\
\text { fuente única }\end{array}$ & $2 .^{\mathrm{a}}$ Fuente & $3 .^{\mathrm{a}}$ Fuente & $\begin{array}{c}1 .^{\mathrm{a}} \text { Fuente o } \\
\text { fuente única }\end{array}$ & 2. $^{\mathrm{a}}$ Fuente & $3 .^{\mathrm{a}}$ Fuente \\
\hline 41,75 & 13,82 & 7,86 & 22,91 & 8,61 & 5,01 \\
\hline
\end{tabular}

Fuente: Elaboración propia.

Atendiendo a este criterio en los distintos medios de comunicación de Granada y en datos globales, las mujeres aparecen como primera fuente en el 22,91 de las informaciones con declaraciones mientras que los hombres consiguen esta posición en el 41,75. Esta situación se repite en las declaraciones que aparecen en segundo lugar ya que las mujeres consiguen esta segunda posición en el 8,61 de las informaciones y los hombres en el 13,82. Para el tercer puesto o sucesivos, el esquema continúa con 
la misma tónica. Los hombres obtienen un 7,86 de apariciones como tercera fuente o sucesivas y las mujeres un 5,01.

\section{TABLA 4}

Posición de las declaraciones de hombres y mujeres en los medios de comunicación de Granada. Porcentajes por formato

\begin{tabular}{|c|c|c|c|c|c|c|}
\hline \multirow[b]{2}{*}{ Medios } & \multicolumn{3}{|c|}{ Número de declaraciones de hombres } & \multicolumn{3}{|c|}{ Número de declaraciones de mujeres } \\
\hline & $\begin{array}{l}\text { 1. }{ }^{\circ} \text { Fuente o } \\
\text { fuente única }\end{array}$ & 2. ${ }^{\circ}$ Fuente & $\begin{array}{c}3 .^{\circ} \text { Fuente o } \\
\text { sucesivas }\end{array}$ & $\begin{array}{l}\text { 1. }{ }^{\circ} \text { Fuente o } \\
\text { fuente única }\end{array}$ & $2 .^{\circ}$ Fuente & $\begin{array}{c}3 .^{\circ} \text { Fuente o } \\
\text { sucesivas }\end{array}$ \\
\hline Radios & 48,61 & 12,50 & 4,61 & 26,00 & 6,48 & 1,80 \\
\hline Televisiones & 27,32 & 17,53 & 13,40 & 17,01 & 11,34 & 13,40 \\
\hline Prensa en papel & 47,62 & 13,29 & 8,03 & 21,30 & 7,01 & 2,75 \\
\hline Prensa digital & 43,83 & 14,19 & 9,25 & 20,99 & 8,65 & 3,09 \\
\hline Agencias & 41,41 & 11,62 & 4,04 & 29,29 & 9,60 & 4,04 \\
\hline
\end{tabular}

Fuente: Elaboración propia.

Una lectura pormenorizada de estos datos nos lleva a constatar que esta diferencia en el número de declaraciones femeninas y masculinas como primera fuente en los periódicos en papel es aún mayor. En este caso, las mujeres constituyen primera fuente informativa en un 21,30 de las informaciones publicadas mientras que los hombres aparecen en un 47,61, lo que arroja un resultado positivo para ellos de más de 26 puntos porcentuales. Esta gran diferencia se repite en los casos en los que sus opiniones son requeridas como segunda fuente ya que ellas recaban el 7,01\% y ellos 13,28\%, casi el doble. Las cifras para la tercera y sucesivas apariciones son de $8,02 \%$ para ellos y un 2,75\% para ellas, es decir, los varones aparecen tres veces más que las mujeres en esta posición.

La prensa digital, aunque renovada en el soporte y en los mensajes, presenta unos resultados muy similares a los diarios en papel. Un total de 22 puntos porcentuales de diferencia separan a hombres y mujeres como personas que plasman sus opiniones en las noticias ya que la cifra para ellos se sitúa en el 43,83 mientras que ellas se quedan en un 20,99. Este desequilibrio se mantiene para la segunda posición de las declaraciones y alcanza el triple en la tercera o sucesivas. Situaciones similares presentan los formatos radiofónicos y las agencias de noticias en sus despachos con informaciones elaboradas aunque en estas últimas las segundas reducen las diferencias y alcanzan el equilibrio en la tercera o sucesiva fuente.

Las menores diferencias se encuentran en las televisiones aunque para la primera fuente se sigue prefiriendo al varón que aparece como voz principal en el 27,32 de 
las informaciones frente al 17,01 de las mujeres, algo más de 10 puntos porcentuales. $\mathrm{Al}$ igual que las agencias, el desequilibrio se reduce para la segunda fuente y se iguala en la tercera.

\subsection{NÚMERO DE FUENTES EN LAS NOTICIAS}

Del total de las 1.586 noticias analizadas, 1.212 se presentan a la audiencia con una única fuente informativa lo que representa el 76,42 de las informaciones. Con dos fuentes se han encontrado 240 que se corresponde con el 15,13, con tres fuentes 81 que representan el 5,11 y con cuatro o más fuentes un total de 53 , lo que representa el 3,34 del total. Los datos reflejan que, en líneas generales, el tratamiento de la prensa de proximidad en Granada ofrece a su audiencia una información carente de profundidad con la que no se logra «completar la historia» (Rodríguez et al., 2015) ya que las fuentes son recursos imprescindibles para construir el relato.

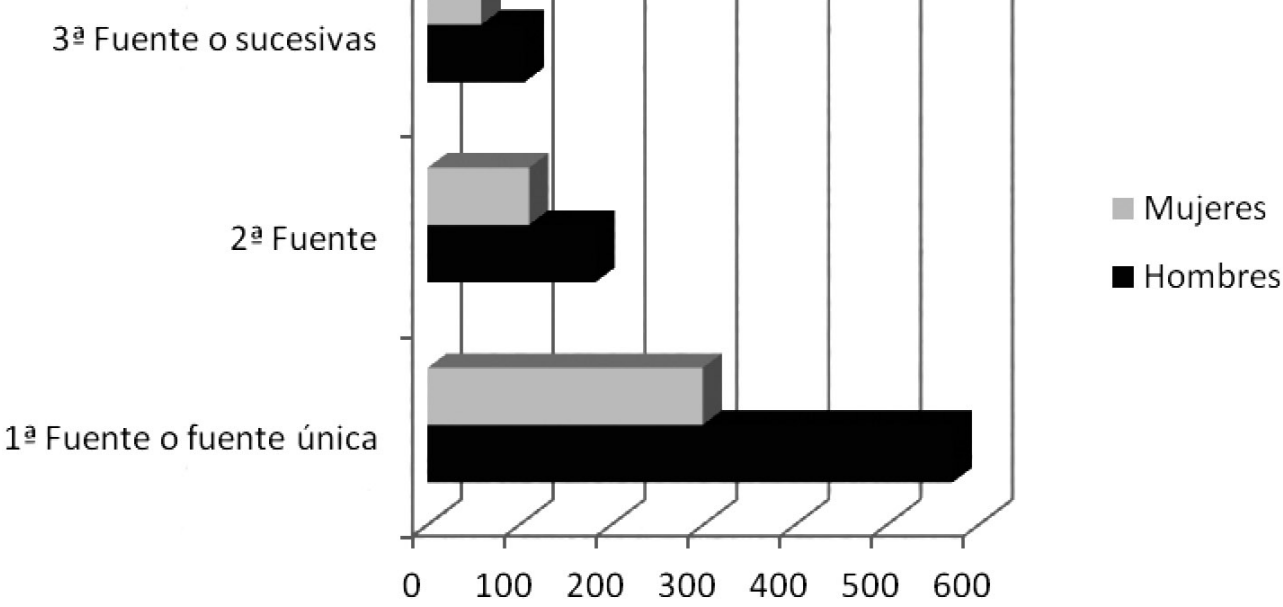

Fuente: Elaboración propia.

GrÁFICO 1

Noticias con declaraciones de hombres y mujeres y posición que ocupan

La lectura atenta de los datos atendiendo al número de voces femeninas y masculinas con declaraciones identificadas en las noticias y el orden que éstas ocupan dentro de las informaciones, confirma que la paridad está aún lejos de alcanzarse. De las 861 citas textuales de varones, 572 aparecen como primera fuente o fuente única, 
183 se localizan como segunda fuente y 106 como tercera o sucesivas. En el caso de las 470 declaraciones identificadas para las mujeres, 300 se realizan como primera o única fuente informativa, 111 como segunda y 59 aparecen en tercer lugar o sucesivos. Como indica la profesora Juana Gallego (2010), «el grupo de hombres y mujeres [de los medios de comunicación] conforman una privilegiada atalaya desde la que contemplan e interpretan el mundo» pero la sociedad ha evolucionado y los discursos deberían adecuarse a los tiempos que vivimos.

\subsection{Análisis de las CONVOCATORias de AGENCIA}

Como indicamos en la metodología de este estudio, además de analizar las informaciones elaboradas por los medios de comunicación y las agencias de noticias con delegación estable en Granada, se evaluaron además las 184 convocatorias de actos informativos distribuidos por estas últimas para observar si el desequilibrio de género tenía su origen en las fuentes informativas señaladas por instituciones, colectivos, partidos políticos, organizaciones empresariales, agentes culturales o sindicatos, entre otros, en el momento de elegir quiénes de sus representantes comparecen ante los medios de comunicación para informar de sus actuaciones a nivel local.

Aunque las convocatorias de las citas informativas no pueden considerarse noticias en sí mismas, configuran el paso intermedio para la elaboración de las informaciones que los medios ofrecerán con posterioridad a la audiencia. La propia disposición de los intervinientes en el anuncio del acto informativo también nos aporta información sobre el peso que cada cual tiene dentro de sus organizaciones. Los gabinetes de prensa, que envían sus convocatorias a las agencias para que éstas conformen la agenda diaria que distribuye a sus abonados, indican el nombre y apellidos de los comparecientes y el lugar que ocupan dentro del orden jerárquico de su organización o empresa, además del asunto sobre el que hablarán ante los medios de comunicación. Por otra parte, en los actos informativos en los que se anuncia la participación de varios intervinientes, el orden en el que aparecen también nos ofrece datos relevantes porque esta secuencia suele ser reproducida por los medios de comunicación en el momento de elaborar sus informaciones. 
TABla 5

Origen de las fuentes en las convocatorias de las agencias de noticias

\begin{tabular}{|l|c|c|}
\hline \multicolumn{1}{|c|}{ Origen de las fuentes informativas } & $\begin{array}{c}\text { Número de } \\
\text { convocatorias }\end{array}$ & $\begin{array}{c}\text { Porcentaje } \\
\text { sobre el total }\end{array}$ \\
\hline Gobierno central & 2 & 1,09 \\
\hline Junta de Andalucía & 41 & 22,28 \\
\hline Diputación Provincial de Granada & 15 & 8,15 \\
\hline Ayuntamiento de Granada & 46 & 25,00 \\
\hline Universidad de Granada & 12 & 6,53 \\
\hline Partidos Políticos & 16 & 8,70 \\
\hline Organizaciones sindicales & 8 & 4,35 \\
\hline Tribunales & 2 & 1,09 \\
\hline Colectivos ciudadanos y ongs & 5 & 2,72 \\
\hline Bancos, cajas y entidades financieras & 1 & 0,54 \\
\hline Organizaciones empresariales & 5 & 2,72 \\
\hline Policía y Guardia Civil & 1 & 0,54 \\
\hline Colegios profesionales & 1 & 0,54 \\
\hline Clubes deportivos & 16 & 0,00 \\
\hline Artistas, escritores y profesionales liberales & 10 & 5,44 \\
\hline Fundaciones y asociaciones culturales & 18 & 0,54 \\
\hline Otros & & 9,77 \\
\hline
\end{tabular}

Fuente: Elaboración propia.

Las instituciones y los partidos políticos dominan la agenda informativa de la información local en Granada. Las convocatorias de la Junta de Andalucía, la Diputación Provincial y el Ayuntamiento representan casi dos tercios de las citas informativas, un 64,13 . En el extremo opuesto se sitúan las organizaciones de la sociedad civil y los temas relacionados con la cultura (fundaciones, colectivos ciudadanos y ONGs, colegios profesionales y sindicatos) que, sumados, alcanzan sólo el 13,59 de las convocatorias. 


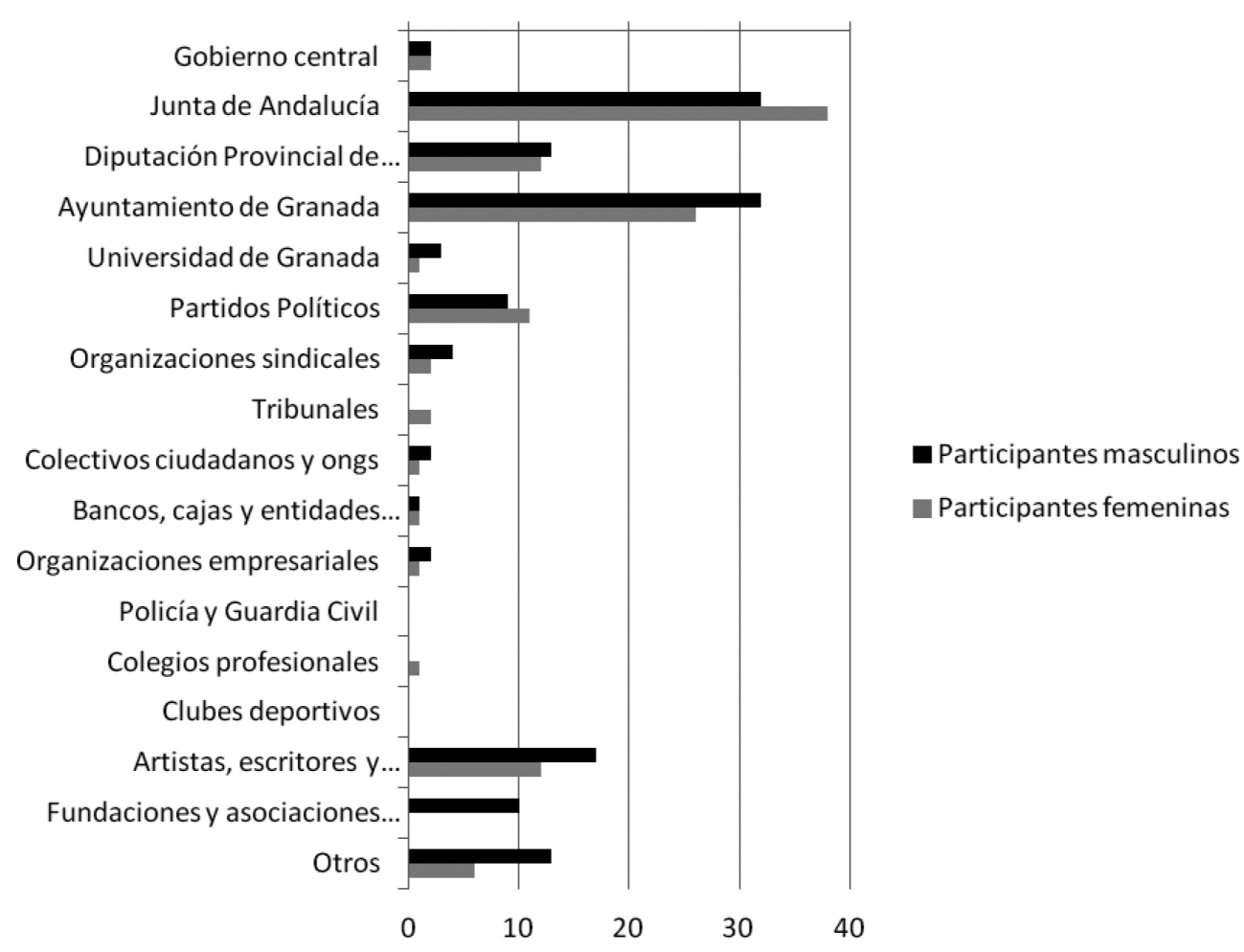

Fuente: Elaboración propia.

\section{GRÁFICO 2}

Intervinientes en las convocatorias de prensa de las agencias de noticias. Distribución por género

Los desequilibrios de género en las convocatorias no aparecen tan acusados, incluso en algunos sectores generadores de noticias en los que se ha dividido la información local de Granada para la elaboración de este estudio, las mujeres aparecen con más frecuencia en las convocatorias que los hombres. Es el caso de las citas informativas anunciadas por la Junta de Andalucía, los partidos políticos, los tribunales o los colegios profesionales. En los encuentros informativos de los medios de comunicación convocados por la Diputación de Granada, prácticamente se alcanza el equilibrio, una equidad conseguida también en los temas relacionados con los bancos, cajas y entidades financieras y en las convocatorias realizadas por representantes del Gobierno Central en la provincia granadina. 
TABLA 6

Orden de las intervenciones en las convocatorias de prensa de las agencias

\begin{tabular}{|l|c|c|c|c|}
\hline \multirow{2}{*}{} & \multicolumn{4}{|c|}{ Número de participantes en las convocatorias } \\
\cline { 2 - 5 } & Interviniente 1 & Interviniente 2 & Interviniente 3 & Interviniente 4 \\
\hline Hombres & $28,91 \%$ & $17,18 \%$ & $7,42 \%$ & $3,91 \%$ \\
\hline Mujeres & $29,30 \%$ & $9,76 \%$ & $3,13 \%$ & $0,39 \%$ \\
\hline
\end{tabular}

Fuente: Elaboración propia.

Contrariamente a lo que ocurre en las informaciones elaboradas, las convocatorias de prensa consiguen el equilibrio de género en el primer lugar de las intervenciones, incluso con un leve repunte de las mujeres. El resto de posiciones dentro de las citas informativas anunciadas no consigue la paridad pero no presenta las diferencias tan abrumadoras que se registran en las informaciones que se ofrecen a la audiencia. Estos datos corroboran un desajuste entre el planteamiento diseñado por las fuentes informativas (instituciones, partidos políticos, organizaciones empresariales...) en el momento de decidir quiénes presentan sus hechos noticiosos a los medios de comunicación de acuerdo con el organigrama de responsabilidades de cada una de ellas y el resultado final de esas informaciones una vez elaboradas por los profesionales del periodismo. El tránsito entre la convocatoria y la noticia abandona las declaraciones de las mujeres como sujetos activos e identificados de las informaciones.

\section{Conclusiones}

Como se ha podido constatar con el análisis de los datos, el desequilibrio de género es una constante en los medios de comunicación de Granada. La prensa de proximidad reproduce los esquemas de la prensa de ámbito nacional y concede mayor protagonismo a las voces masculinas que aparecen identificadas con nombres, apellidos y cargo casi en el doble de ocasiones que las voces femeninas. Esta situación se repite en todos los soportes analizados (emisoras de radio, televisiones, diarios en papel, prensa digital y agencias de noticias en sus teletipos con información elaborada) por lo que nuestra hipótesis de partida queda reforzada.

Sobre la posición de las declaraciones textuales de hombres y mujeres en los medios de comunicación granadinos, el análisis de las 1586 noticias evaluadas confirma que las opiniones y valoraciones realizadas por mujeres quedan relegadas a posiciones con menos relevancia informativa $y$, aun en estas posiciones secundarias, la presencia de las citas textuales de los varones se localizan en un mayor porcentaje que las de las mujeres. Especialmente significativas son las diferencias registradas 
en los diarios en papel que alcanzan los 26 puntos porcentuales; la prensa digital ha adoptado los mismos esquemas que la prensa tradicional y presenta un desequilibrio a favor de los varones en 23 puntos. El formato que más se acerca a la paridad es la televisión a pesar de los 10 puntos favorables a ellos.

El análisis de los temas abordados en las noticias nos confirma que el peso de la información institucional es muy elevado en los medios de proximidad y copa más de la mitad de la agenda informativa. La presencia de mujeres en estos organismos facilita su inclusión en las noticias pero los hombres continúan dominando este espacio público a través de la información publicada y la presencia de declaraciones identificadas en los medios de comunicación obtiene porcentajes más elevados. Incluso en aquellas instituciones granadinas en cuya cúspide se sitúan mujeres - la Delegación del Gobierno de la Junta de Andalucía y la Universidad de Granada- el número de voces masculinas supera al de voces femeninas.

Los resultados obtenidos a partir de las convocatorias de agencia obligan a una reflexión profunda de los medios de comunicación y sus prácticas en el momento de diseñar la agenda diaria. El equilibrio de hombres y mujeres como primera fuente informativa en las convocatorias no se traduce posteriormente en las noticias elaboradas para su difusión por los medios. El tránsito de la convocatoria a la información redactada relega a las mujeres o las oculta.

Es destacable también la ausencia de voces con identidades de género diferentes al eje binario masculino/femenino lo que pone de manifiesto el alejamiento de los medios de comunicación de la realidad social en la que se asientan. Los colectivos de personas gays, lesbianas, bisexuales, transexuales e intersexuales se encuentran completamente silenciados y sin espacio en los medios lo que refuerza el diseño profundamente masculinizado de la agenda.

En resumen, la perspectiva de género en los medios de comunicación se presume urgente en un momento en el que la sociedad está registrando cambios significativos. El pasado 8 de marzo, las profesionales de los medios de comunicación nos sumamos con fuerza a las reivindicaciones feministas con el movimiento Las Periodistas Paramos (LPP), una iniciativa que se mantiene activa y con el que estamos decididas a desligarnos de la cultura machista de las redacciones. Los resultados de esta acción reivindicativa deberán tener su reflejo en el tratamiento de las informaciones por lo que sería aconsejable realizar una nueva revisión de la prensa de proximidad en un futuro cercano para comprobar su incidencia. 


\section{Referencias bibliográficas}

Altés, E. y Gallego, J. (2004). Estereotipos y roles de género en los medios de comunicación. En LÓPEZ DÍAZ, Pilar (ed.), Manual de información en género (pp. 37-69). Madrid: Instituto Oficial de Radio y Televisión.

Beltrán Vidal, J.M. (2014). Género, Constitución y medios de comunicación. En VV.AA., Igualdad y democracia: el género como categoría de análisis jurídico. Estudios en homenaje a la profesora Julia Sevilla Merino (pp.718-730). Valencia: Corts Valencianes.

Blanco Castilla, E. (2005). Mujer y poder en los medios: Dificultades para una incorporación plena. En Informe anual de la profesión periodística 2005. Madrid: Asociación de la prensa.

Canel, M.J. (2000). Periodistas al Descubierto. Madrid: Centro de Investigaciones Sociológicas.

Caro González, F.J., García-Gordillo, M. y Bezunartea Valencia, O. (2010). Las mujeres como sujeto de la información: techo de cristal y audiencias en las empresas periodísticas. En Vázquez Bermúdez, I. (coord.), Investigaciones multidisciplinares en género: II Congreso Universitario Nacional Investigación y Género (pp. 187-197). Sevilla, 17 y 18 de junio.

Casero, A. (2012). El periodismo político en España: algunas características definitorias. Periodismo político en España: concepciones, tensiones y elecciones (pp. 19-46). La Laguna: Sociedad Latina de Comunicación Social. Recuperado de http://hdl.handle. net/10234/80553

Courteney, A. y Lockeretz, S. (1971). A woman's place: an analysi of the roles portayed by women in magazine advertisements. Journal of Marketing Research, 8. (1) 92-95.

Culley, J.D. y Bennett, R. (1976). Selling women, selling Blacks. Journal of Communication, 26, 160-174.

Faoaga, C. y Secanella, P.M. (1984). Umbral de presencia de las mujeres en la prensa española. Madrid: Instituto de la Mujer.

Franquet, R. (1982a). Por un discurso no androcéntrico de los medios de comunicación. En VV.AA. El sexismo en la ciencia. Barcelona: Universidad Autónoma de Barcelona.

Franquet, R. (1982b). La mujer y su relación con los medios. Chasqui: Revista Latinoamericana de Comunicación (4 julio-septiembre) 36-43 Recuperado de http://revistachasqui. org/index.php/chasqui/article/view/1697/1717

Gallagher, M. (1983). Unequal opportunities. The case of women and Media. París: Unesco.

Gallego, J. (2002). La prensa por dentro. Producción informativa y transmisión de estereotipos de género. Barcelona: Libros de la Frontera.

Gallego J. (2003). Producción informativa y transmisión de estereotipos de género en la prensa diaria. Comunicación y sociedad, XVI (2), 49-66. Recuperado de http://hdl. handle.net/10171/8055

Gallego, J. (2010). De reinas a ciudadanas. Género, comunicación y cambio social. II Jornadas Mujer y Medios de Comunicación (pp. 5-28). Universidad de País Vasco. 
García de Cortázar, M. y García de León, M.A. (coords.) (2000). Profesionales del Periodismo. Hombres y Mujeres en los Medios de Comunicación. Colección monografias, n. ${ }^{\circ} 181$. Madrid: Centro de Investigaciones Sociológicas.

García Gordillo, M.; Bezunartea Valencia, O.; Rodríguez Rey, A. y Sánchez González, M.T. (2014). La calidad en los medios y el uso de fuentes periodísticas. Una evolución del VAP (pp. 1.541-1.555). IV Congreso Internacional de la Asociación Española de Investigación en Comunicación.

Global Alliance on Media and Gender. Recuperado de http://gamag.net/

International Women's Media Foundation (IWMF) (2011) Global Report on the Status of Women in the News Media. Washington: Unesco. Recuperado de https://www.iwmf. org/wp-content/uploads/2018/06/IWMF-Global-Report.pdf

Humanes, M.L. (2003). Evolución de Roles y Actitudes. Cultura y Modelos Profesionales del Periodismo. Telos, (54 enero-marzo). Recuperado de https://telos.fundacion telefonica.com/archivo/numero054/cultura-y-modelos-profesionales-del-periodismo/

Asociación de la Prensa de Madrid. Informe anual de la profesión periodística en España 2017. Recuperado de https://www.apmadrid.es/publicaciones/informe-anual-de-laprofesion/

Karpf, A. (1980). Women and radio. Women's Studies Internationnal Quarterly, 3 (1) 41-54.

Lichter, R., Lichter, L.S. y Rothman, S. (1986). From Lucy to Lacey: TV's Dream Girls. Public Opinion, (9) 16-19.

Melin-Higgins, M. y Djerf Pierre, M. (1998). Networking in newsroom. Journalist and Gender Cultures. Paper of AIERI. Glasgow.

Moreno Sardá, A. (2001). Reflejos de los temas de género en los medios de comunicación: un asunto controvertido. En Caporale Bizzini, S.; Fuentes Soriano, O.; Mañas Viejo, C.; Montesinos Sánchez, M.N. y Ruiz Cantero, M.T. (eds.). La difusión del conocimiento en los estudios de las mujeres. Dinámicas y estrategias de poder y ciudadanía. Actas del II Congreso Internacional de AUDEM (pp. 151-166). Universidad de Alicante 7, 8 y 9 de mayo de 2001. Centro de Estudios sobre la Mujer.

Moreno Sardà, A. y Simelio Sola, N. (2011). El periodismo margina a mujeres y hombres como protagonistas de la democracia. Resultados preliminares de la evaluación de la presencia que las personas reciben en los titulares de El País, 2005 y 2010. Actas del III Congreso Latina de Comunicación Social, diciembre 2011. Universidad de La Laguna.

Moreno Sardà, A.; Simelio Solà, N.; Rovetto Gonem, F. y Buitrago Londoño, A. (2007). Periodismo y ciudadanía plural: problemas, rutinas y retos. Estudios sobre el Mensaje Periodístico, 13 157-168. Recuperado de https://revistas.ucm.es/index.php/ESMP/issue/ view/ESMP070711/showToc

Papí Gálvez, N. (2003). Un nuevo paradigma para el análisis de las redes sociales: el enfoque de género. Feminismo/s, 1, 135-148 Recuperado de: https://rua.ua.es/dspace/ bitstream/10045/2866/1/Feminismos_1_10.pdf

Pearson, J.C.; Turner, L.H. y Todd-Mancillas, W. (1993). Comunicación y género. Barcelona: Paidós. 
Report of the Fourth World Conference on Women. Beijing, 4-15 september 1995. Recuperado de http://www.un.org/womenwatch/daw/beijing/pdf/Beijing\%20full\%20 report $\% 20 \mathrm{E} . \mathrm{pdf}$

Rodríguez Rey, A.; Enguix González, A.; Rojas Torrijos, J.L. y García Gordillo, M. (2015). La calidad de los medios y el uso de fuentes periodísticas en la prensa local de referencia en España. Estudios sobre el Mensaje Periodístico, 21, (número especial noviembre) 85-100. doi: 10.5209/rev_ESMP.2015.v21.50659

Schudson, M. (2003). The Sociology of News. New York: Norton.

Soriano, J.; Cantón, M.J. y Díez, M. (2005). La pseudofeminización de la profesión periodística en España. Zer Revista de Estudios de Comunicación 10 (19) 35-52. Recuperado de http://www.ehu.eus/ojs/index.php/Zer/article/view/3838/3420

Tan, A. (1982). Television use and social stereotypes. Journalism Quaterly, 59, 119-122.

The Global Media Monitoring Project (GMMP) http://whomakesthenews.org/gmmp-2015

Trancart, M. (1999). Les femmes absences de l'information. Quand les chiffres parlent. En Rapport sur les recherches dans l'Unión Européenne, Emploi \& Affaires Sociales. Bruselas: Comisión Europea.

Tuchman, G. (1993). Realism and romance: The study of media effects. Journal of Communication, 43 (4) 36-41.

Unesco UnitWin Network On Gender, Media and ICTs. Recuperado de http://www. unitwin.net/

Vera Balanza, T. (2009). El perfil de las periodistas en Andalucía: formación y expectativas laborales. Un balance, 1989-2009. En Núñez Domínguez, T. y Loscertales Abril, F. (coords.) Las mujeres y los medios de comunicación. Una mirada de veinte años (1989-2009) (pp. 57-73) Sevilla: Instituto Andaluz de la Mujer.

Zemach, T. y Cohen, A.A. (1986). Perception of gender and quality. Journal of Broadcasting and Electronic Media, 30, 427-444.

Zoonen, L. van (1994). Feminist Media Studies. London:Sage. 